October 2001

\title{
De Sitter space versus Nariai Black Hole: stability in d5 higher derivative gravity.
}

\author{
SHIN'ICHI NOJIRI and SERgEI D. ODINTSOV $\$$ Z \\ Department of Applied Physics \\ National Defence Academy, Hashirimizu Yokosuka 239-8686, JAPAN \\ - Instituto de Fisica de la Universidad de Guanajuato, \\ Lomas del Bosque 103, Apdo. Postal E-143, 37150 Leon,Gto., MEXICO
}

\begin{abstract}
d5 higher derivative gravity on the Schwarzschild-de Sitter (SdS) black hole background is considered. Two horizons SdS BHs are not in thermal equilibrium and Hawking-Page phase transitions are not expected there, unlike to the case of AdS BHs. It is demonstrated that there exists the regime of d5 theory where Nariai BH which is extremal limit of SdS BH is stable. It is in the contrast with Einstein gravity on such background where only pure de Sitter space is always stable. Speculating on the applications in proposed dS/CFT correspondence, these two (de Sitter and Nariai) stable spaces may correspond to confining-deconfining phases in dual CFT.
\end{abstract}

PACS numbers: 04.50.+h, 04.70.Dy, 11.25.Db

\footnotetext{
${ }^{1}$ nojiri@cc.nda.ac.jp

${ }^{2}$ On leave from Tomsk State Pedagogical University, 634041 Tomsk, RUSSIA. odintsov@ifug5.ugto.mx, odintsov@mail.tomsknet.ru
} 
The increasing evidence indicating a positive cosmological constant for our universe calls to better study of de Sitter gravity. One of the fundamental questions in de Sitter gravity is related with holographic principle which is presumably realized there in the form of $\mathrm{dS} / \mathrm{CFT}$ correspondence suggested in ref.[1] (for earlier attempts on dS/CFT duality see [2]). Despite the number of efforts [3, 4] (for a recent review, see [5]) still dS/CFT correspondence is not understood on the same level as AdS/CFT correspondence. Moreover, the corresponding consistent dual CFT is not yet formulated. Nevertheless, the hunt for $\mathrm{dS} / \mathrm{CFT}$ correspondence continues. It is expected that analogy with AdS/CFT may be often helpful in such investigation.

It has been observed quite long ago by Hawking and Page [6] that AdS black hole $(\mathrm{BH})$ thermodynamics admits phase transitions in the following way: low temperature BHs are not stable and they decay into global AdS space. High temperature BHs are more stable than global AdS space. Hawking-Page phase transitions for $5 \mathrm{~d}$ AdS BHs are very important in frames of AdS/CFT. Indeed, they were interpreted by Witten [7] as corresponding to a deconfinement-confinement transition in the large- $N$ limit of an $\mathcal{N}=4$ $S U(N)$ super Yang-Mills theory living on the boundary of $5 \mathrm{~d}$ AdS BH.

The interesting question appears: Can the similar effect be expected for proposed dS/CFT correspondence? From first look it seems that the answer is completely negative. The reason is that there are two Hawking temperatures in Schwarzschild-de Sitter (SdS) BH (which is the natural analog of AdS $\mathrm{BH})$ since there are two horizons, black hole one and cosmological one. Then $\mathrm{SdS}$ spacetime is not in the thermal equilibrium unlike to AdS BH. Moreover, it is known that pure de Sitter space is the only stable space among SdS backgrounds for Einstein gravity. There is, however, second exception. It is the Nariai space, which is the extremal limit where the black hole horizon coincides with the cosmological one.

In the present Letter we discuss the question of stability of dS and Nariai $\mathrm{BH}$ in higher derivative gravity (for general introduction to such theory, see [8]). We will show that despite the absence of Hawking-Page phase transitions, there is some theory regime (defined by coefficients of higher derivative terms) where Nariai BH is stable and does not decay into pure de Sitter space. Brief speculation of the relevance of this observation to proposed $\mathrm{dS} / \mathrm{CFT}$ correspondence is made. 
The general action of $d+1$ dimensional $R^{2}$-gravity is given by

$$
S=\int d^{d+1} x \sqrt{-\hat{G}}\left\{a \hat{R}^{2}+b \hat{R}_{\mu \nu} \hat{R}^{\mu \nu}+c \hat{R}_{\mu \nu \xi \sigma} \hat{R}^{\mu \nu \xi \sigma}+\frac{1}{\kappa^{2}} \hat{R}-\Lambda\right\} .
$$

For simplicity, we only consider the $c=0$ case, for a while. Then Schwarzschild-anti de Sitter or Schwarzschild-de Sitter spacetime is an exact solution:

$$
\begin{aligned}
& d s^{2}=\hat{G}_{\mu \nu} d x^{\mu} d x^{\nu}=-\mathrm{e}^{2 \rho_{0}} d t^{2}+\mathrm{e}^{-2 \rho_{0}} d r^{2}+r^{2} \sum_{i, j}^{d-1} g_{i j} d x^{i} d x^{j} \\
& \mathrm{e}^{2 \rho_{0}}=\frac{1}{r^{d-2}}\left(-\mu+\frac{k r^{d-2}}{d-2}+\frac{r^{d}}{L}\right) .
\end{aligned}
$$

Here $g_{i j}$ is the metric of the $(d-1)$-dimensional Einstein manifold, which is defined by $R_{i j}=k g_{i j}$, where $R_{i j}$ is the Ricci tensor defined by $g_{i j}$ and $k$ is a constant. For example, we have $k>0$ for the sphere, $k<0$ for the hyperboloid, and as a special case, flat space for $k=0$. The parameter $L$ in (2) is related with the length parameter of the asymptotic AdS or dS spacetime and is found solving the equation

$$
0=\frac{d^{2}(d+1)(d-3) a}{L^{2}}+\frac{d^{2}(d-3) b}{L^{2}}-\frac{d(d-1)}{\kappa^{2} L}-\Lambda
$$

If $L>0$, the spacetime is SAdS and if $L<0, \operatorname{SdS}$. Here we consider the case of $L<0$ and $d=4$. In dS, we cannot embedd the hyperbolic or the flat space as the surface with constant $r$. Then one only discusses the case that $g_{i j}(2)$ is the metric of the unit sphere

$$
\sum_{i, j}^{3} g_{i j} d x^{i} d x^{j}=d \Omega_{3}^{2}
$$

In this case $k=2$. Defining the length parameter $l$ by

$$
l^{2}=-L,
$$

$\mathrm{e}^{2 \rho}$ in the metric (2) has the following form

$$
\mathrm{e}^{2 \rho_{0}}=\frac{1}{r^{d-2}}\left(-\mu+r^{2}-\frac{r^{4}}{l^{2}}\right)
$$


Then there are two horizons, where $\mathrm{e}^{2 \rho_{0}}=0$, at

$$
r=r_{c, b h}^{2} \equiv \frac{l^{2} \pm \sqrt{l^{4}-4 \mu l^{2}}}{2}
$$

where the plus sign corresponds to the cosmological horizon $r=r_{c}$ and the minus to the black hole one $r=r_{b h}$. The corresponding Hawking temperature $T_{H}$ is given by

$$
T_{H}=\frac{1}{4 \pi}\left|\frac{d\left(\mathrm{e}^{2 \rho_{0}}\right)}{d r}\right|_{r=r_{c, b h}}\left|=\frac{1}{2 \pi}\right| \frac{\mu}{r_{c, b h}^{3}}-\frac{r_{c, b h}}{l^{2}} \mid .
$$

For the pure dS case, where $\mu=0$, we find

$$
r_{b h}=0, \quad r_{c}=l, \quad T_{H}=\frac{1}{2 \pi l}
$$

and for the Nariai space [9], where $\mu=\frac{l^{2}}{4}$,

$$
r_{b h}=r_{c}=\frac{l}{\sqrt{2}}, \quad T_{H}=\frac{15}{2 \sqrt{2} l \pi} .
$$

We should note that when $c \neq 0$ in (1), the general Schwarzschild-(anti) de Sitter spacetime is not the exact solution. Nevertheless, even if $c \neq 0$, the pure dS and the Nariai space are exact solutions since the Riemann curvature is covariantly constant:

$$
\hat{R}_{\mu \nu \xi \sigma}=\frac{1}{l^{2}}\left(\hat{G}_{\mu \xi} \hat{G}_{\nu \sigma}-\hat{G}_{\mu \sigma} \hat{G}_{\nu \xi}\right) .
$$

Here, instead of (3), the length parameter $l^{2}$ is given by

$$
\begin{aligned}
0= & \frac{a}{l^{4}}(d+1) d^{2}(d-3)+\frac{b}{l^{4}} d^{2}(d-3) \\
& +\frac{2 c}{l^{4}} d(d-3)+\frac{d(d-1)}{\kappa^{2} l^{2}}-\Lambda .
\end{aligned}
$$

Then in what follows we also consider the case of $c \neq 0$.

Before going forward, we give some remarks about the Nariai space, which is given in the Nariai limit $\mu \rightarrow \frac{l^{2}}{4}$. Before taking the limit, one changes the coordinate $(t, r)$ to $(\tau, \theta)$ by

$$
t=\frac{l}{\sqrt{l^{2}-4 \mu}} \tau, \quad r^{2}=\frac{l^{2}}{2}-\cos \theta \frac{\sqrt{l^{4}-4 \mu l^{2}}}{2} .
$$


Then the black hole horizon corresponds to $\theta=0$ and the cosmological one to $\theta=\pi$. In the coordinates $(\tau, \theta)$, the metric of SdS is rewritten by

$$
d s^{2}=-\frac{\sin ^{2} \theta}{2\left(l^{2}-\cos \theta \sqrt{l^{4}-4 \mu l^{2}}\right)} d \tau^{2}+\frac{l^{2}}{2} d \theta^{2}+\left(\frac{l^{2}}{2}-\cos \theta \sqrt{l^{4}-4 \mu l^{2}}\right) d \Omega_{3}^{2} .
$$

Then by taking the Nariai limit $\mu \rightarrow \frac{l^{2}}{4}$, one finds

$$
d s_{\text {Nariai }}^{2}=-\frac{\sin ^{2} \theta}{2} d \tau^{2}+\frac{l^{2}}{2} d \theta^{2}+\frac{l^{2}}{2} d \Omega_{3}^{2} .
$$

If we Wick-rotate the time coordinate $\tau$ by

$$
\tau \rightarrow i l \tilde{\tau}
$$

the metric (15) is the direct product of $S_{2}$ and $S_{3}$ with the radius $\frac{l}{\sqrt{2}}$ and we find $\tilde{\tau}$ should have the periodicity of $2 \pi$.

In 4 dimensional $(d=3)$ Nariai space, there occurs a very interesting phenomenon called "anti-evaporation", which was first found in [10] by using $2 \mathrm{~d}$ trace anomaly induced effective action including dilaton [11, 12, 13]. It corresponds to quantum expansion of $\mathrm{BH}$. (It is not yet completely clear if this is fundamental or just transitionary effect). This phenomenon has been confirmed by using 4d trace anomaly induced effective action [14].

Let us discuss the free energies of the pure dS and the Nariai space. Since for these cases, the scalar, Ricci and Riemann curvatures are given by

$$
\hat{R}=\frac{20}{l^{2}}, \quad \hat{R}_{\mu \nu}=\frac{4}{l^{2}} G_{\mu \nu}, \quad \hat{R}_{\mu \nu \xi \sigma}=\frac{1}{l^{2}}\left(\hat{G}_{\mu \xi} \hat{G}_{\nu \sigma}-\hat{G}_{\mu \sigma} \hat{G}_{\nu \xi}\right) .
$$

the action (11) with $d=4$ is given by

$$
\begin{aligned}
S & =\left(\frac{400 a}{l^{4}}+\frac{80 b}{l^{4}}+\frac{40 c}{l^{4}}+\frac{20}{\kappa^{2}}-\Lambda\right) \int d^{5} x \sqrt{-\hat{G}} \\
& =\left(\frac{320 a}{l^{4}}+\frac{64 b}{l^{4}}+\frac{32 c}{l^{4}}+\frac{8}{l^{2} \kappa^{2}}\right) \int d^{5} x \sqrt{\hat{G}}
\end{aligned}
$$

In the second line of (18), Eq. (3) is used. For pure de Sitter space, one gets

$$
V_{\mathrm{dS}}=\int d^{5} x \sqrt{\hat{G}}=\frac{V_{3}}{T_{H}} \int_{0}^{r_{c}=l} d r r^{3}=\frac{V_{3} l^{4}}{4 T_{H}}=\frac{V_{3}}{4 T_{H}\left(2 \pi T_{H}\right)^{4}},
$$


and for the Nariai space

$$
V_{\text {Nariai }}=\int d^{5} x \sqrt{\hat{G}}=V_{2} V_{3}\left(\frac{l^{2}}{2}\right)^{\frac{5}{2}}=\frac{8 \cdot 15^{5}}{(2 \pi)^{\frac{5}{2}}} \frac{V_{3}}{4 T_{H}\left(2 \pi T_{H}\right)^{4}},
$$

Here $V_{2}=4 \pi$ and $V_{3}$ are the volumes of 2 sphere and 3 sphere, respectively. In (20) it is assumed $\tilde{\tau}$ in (16) has the period $2 \pi$. As it follows from (13) and (16)

$$
i \tilde{\tau}=\frac{t \sqrt{l^{2}-4 \mu}}{l^{2}} .
$$

Then if $t$ has the periodicity of $\frac{1}{T_{H}}$ (near the Nariai limit) when Wick-rotated, the periodicity $\tilde{P}$ of $\tilde{\tau}$ should be

$$
\tilde{P}=\frac{\sqrt{l^{2}-4 \mu}}{T_{H} l^{2}}
$$

Then the volume $V_{\text {Nariai }}$ of the Nariai space in (20) should be modified as

$$
V_{\text {Nariai }} \rightarrow \frac{\tilde{P}}{2 \pi} V_{\text {Nariai }}
$$

which, we should note, vanishes in the Nariai limit $\mu \rightarrow \frac{l^{2}}{4}$ since $\tilde{P}$ vanishes.

Using (18) and (19), we find that the free energy $F=-T_{H} S$ for the pure $\mathrm{dS}$ is given by

$$
F_{\mathrm{dS}}=-\left(\frac{320 a}{l^{4}}+\frac{64 b}{l^{4}}+\frac{32 c}{l^{4}}+\frac{8}{l^{2} \kappa^{2}}\right) \frac{V_{3}}{4\left(2 \pi T_{H}\right)^{4}},
$$

Using (18), (20) and (23), one gets the free energy of the Nariai space:

$$
F_{\text {Nariai }}=-\left(\frac{320 a}{l^{4}}+\frac{64 b}{l^{4}}+\frac{32 c}{l^{4}}+\frac{8}{l^{2} \kappa^{2}}\right) \frac{8 \cdot 15^{5}}{(2 \pi)^{\frac{5}{2}}} \frac{V_{3}}{4\left(2 \pi T_{H}\right)^{4}} \frac{\tilde{P}}{2 \pi} \stackrel{\mu \rightarrow \frac{l^{2}}{4}}{\longrightarrow} 0 .
$$

Therefore the pure $\mathrm{dS}$ is more stable than the Nariai space if

$$
\frac{320 a}{l^{4}}+\frac{64 b}{l^{4}}+\frac{32 c}{l^{4}}+\frac{8}{l^{2} \kappa^{2}}>0
$$


but the Nariai space becomes stable if

$$
\frac{320 a}{l^{4}}+\frac{64 b}{l^{4}}+\frac{32 c}{l^{4}}+\frac{8}{l^{2} \kappa^{2}}<0 \text {. }
$$

Therefore there is a critical point (or surface) at

$$
\frac{320 a}{l^{4}}+\frac{64 b}{l^{4}}+\frac{32 c}{l^{4}}+\frac{8}{l^{2} \kappa^{2}}=0 .
$$

It is easily seen that for pure Einstein gravity, the above equation has no solution. Hence, dS space is always stable there!

The expressions of the free energies (24) and (25) seem to be strange since the fourth inverse power of the temperature appears in the expressions. As we are considering the limits, Nariai limit and the vanishing mass limit, the temperature does not depend on the black hole mass but only depends on the length parameter $l$ as in (9) and (10). The parameter $l$ is not the dynamical variable. Of course, when we include the scalar fields $\phi_{i}$ with potential $V\left(\phi_{i}\right)$, the length parameter can be regarded as a dynamical variable by replacing the cosmological constant $\Lambda$ in the action (1) by the potential $\Lambda \rightarrow V\left(\phi_{i}\right)$. One can also consider the condensation of the anti-symmetric tensor fields as in the usual $\mathrm{AdS}_{5} / \mathrm{CFT}_{4}$ scenario. If $l$ is not the dynamical variable, we should consider the radius of the black hole as a dynamical variable but when the radius changes from one of the (Nariai and the vanishing mass) limits, the system is not in the thermal equilibrium. Then one cannot define the heat capacity and (or) to use the other thermodynamical stability conditions as those developed in ref. 15. This makes difficult to argue about the local thermal stability. One can conjecture that two limits are connected with each other by some thermal inequilibrium process.

From the viewpoint of the WKB approximation of the path integral, the partition function $Z$ can be given by the classical action $S_{\mathrm{cl}}$, where the classical solution is substitued into the action (1),

$$
Z \sim \mathrm{e}^{S_{\mathrm{cl}}} .
$$

The expression (25) is valid even if the system is not in the thermal equilibrium. For the general SdS spacetime, one gets

$$
V_{\mathrm{SdS}}=\int d^{5} x \sqrt{-\hat{G}}=\frac{V_{3}}{T_{H}} \int_{r_{b h}}^{r_{c}} d r r^{3}=\frac{V_{3} l^{4}}{4 T_{H}} \sqrt{1-\frac{4 \mu}{l^{2}}} .
$$


Here we assume the time variable $t$ has a period $\frac{1}{T_{H}}$ although the system is in the thermal inequilibrium. When $c=0$, the action $S_{\mathrm{SdS}}$ for $\mathrm{SdS}$ is also given by $(18)$

$$
S_{\mathrm{SdS}}=\left(\frac{320 a}{l^{4}}+\frac{64 b}{l^{4}}+\frac{8}{l^{2} \kappa^{2}}\right) V_{\mathrm{SdS}} .
$$

The actions for the pure $\mathrm{dS}$ and Nariai space are just given by replacing $V_{\mathrm{SdS}}$ with $V_{\mathrm{dS}}$ in (19) and $\frac{\tilde{P}}{2 \pi} V_{\text {Nariai }}$ in (20) and (23), respectively. Since $\sqrt{1-\frac{4 \mu}{l^{2}}}<1$, we find

$$
V_{\mathrm{dS}}>V_{\mathrm{SdS}}>\frac{\tilde{P}}{2 \pi} V_{\text {Nariai }}=0
$$

Then the classical action for dS is larger than that for SdS if the condition (26) is satisfied. Therefore $\mathrm{dS}$ is stable even locally. On the other hand, if the condition (27) is satisfied, the classical action for the Nariai space is larger than that for SdS. Then the Nariai space becomes stable even locally. Hence, we demonstrated that there is regime of $\mathrm{d} 5$ higher derivative gravity where Nariai BH does not decay into pure dS space.

In case of $\mathrm{AdS}_{5} / \mathrm{CFT}_{4}$ correspondence higher derivative terms like $R^{2}$ terms appear as next-to-leading, $\frac{1}{N}$ correction. The $\mathcal{N}=2$ theory with the gauge group $S p(N)$ arises as the low-energy theory on the world volume on $N$ D3-branes sitting inside 8 D7-branes at an O7-plane [16]. The string theory dual to this theory has been conjectured to be type IIB string theory on $\mathrm{AdS}_{5} \times \mathrm{X}^{5}$ where $\mathrm{X}_{5}=\mathrm{S}^{5} / Z_{2}$ [17], whose low energy effective action is given by

$$
S=\int_{\mathrm{AdS}_{5}} d^{5} x \sqrt{G}\left\{\frac{N^{2}}{4 \pi^{2}} R-\Lambda+\frac{6 N}{24 \cdot 16 \pi^{2}} R_{\mu \nu \rho \sigma} R^{\mu \nu \rho \sigma}\right\} .
$$

Then $R^{2}$-term appears as $1 / N$ correction. We should note that the coupling constants are chosen to be dimensionless by the proper redefinitions. Then one can identify

$$
\frac{1}{\kappa^{2}}=\frac{N^{2}}{4 \pi^{2}}, \quad c=\frac{6 N}{24 \cdot 16 \pi^{2}},
$$

In the model (37), $\Lambda$ is negative and the spacetime is always asymptotically AdS. 
Here as a toy model, we consider the case that $\Lambda$ is positive and is given by

$$
\Lambda=\frac{12 N^{2}}{4 \pi^{2}}-\frac{6 N}{24 \cdot 16 \pi^{2}}
$$

Suppose that $c$ is negative:

$$
\frac{1}{\kappa^{2}}=\frac{N^{2}}{4 \pi^{2}}, \quad c=-\frac{6 N}{24 \cdot 16 \pi^{2}},
$$

It has been demonstrated in ref. [4] that in frames of dS/CFT correspondence such de Sitter higher derivative gravity reproduces the holographic conformal anomaly for above $S p(N)$ super Yang-Mills theory. Then Eq.(12) tells $l^{2}=1$ and Eq.(28) gives the critical point at

$$
N=\frac{1}{4}
$$

Then from Eq.(26), the pure de Sitter space is stable when $N \geq 1$. Of course, since this model is not realistic dual CFT model one can expect that there will be and $\mathrm{dS}$ and Nariai phases when realistic dual CFT will be proposed. (Moreover, even in above model the next powers of the curvatures may qualitatively change the situation). Then presumably one of these two phases will correspond to confinement, while another one to deconfinement in dual CFT. In any case, the fact that Nariai BH may be preferrable vacuum state for some dS gravitational theory looks quite attractive.

Acknoweledgements. We thank M. Cvetič and V. Tkach for helpful discussion. The research by SDO has been supported in part by CONACyT (CP, Ref.990356).

\section{References}

[1] A. Strominger, hep-th/0106113.

[2] C.M. Hull,JHEP 9807 (1998) 021, hep-th/9806146; R. Bousso, JHEP 9906 (1999) 028, hep-th/9906022; E. Witten, hep-th/0106109; A. Volovich, hep-th/0101176; V. Balasubramanian, P. Horava and D. Minic, JHEP 0105 (2001) 043, hep-th/0103171; 
[3] P. Mazur and E. Mottola, hep-th/0106151; M. Li, hep-th/0106184; S. Nojiri and S.D. Odintsov, hep-th/0106191; hep-th/0107134; D. Klemm, hep-th/0106247; Y. Gao, hep-th/0107067; T. Shiromizu, D. Ida and T. Torii, hep-th/0109057; S. Cacciatori and D. Klemm, hep-th/0110031.

[4] S. Nojiri, S.D. Odintsov and S. Ogushi, hep-th/0108172, to appear in Phys.Rev. D.

[5] M. Spradlin, A. Strominger and A. Volovich, hep-th/0110007.

[6] S.W. Hawking and D.N. Page, Comm.Math.Phys. 87 (1983) 577.

[7] E. Witten, Adv.Theor.Math.Phys. 2 (1998) 505.

[8] I.L. Buchbinder, S.D. Odintsov and I.L. Shapiro, Effective Action in Quantum Gravity, IOP Publishing, Bristol and Philadelphia, 1992.

[9] H. Nariai, Sci.Rep.Tohoku Univ.Ser.I 35 (1951) 62.

[10] R. Bousso and S.W. Hawking, Phys.Rev. D57 (1998) 2436; R. Bousso, Phys.Rev. D60 (1999) 063503.

[11] R. Bousso and S.W. Hawking, Phys.Rev. D56 (1997) 7788, hepth/9705236.

[12] S. Nojiri and S.D. Odintsov, Mod.Phys.Lett. A12 (1997) 2083, hepth/9706009; Phys.Rev. D57 (1998) 2363, hep-th/9706143; for a review, see S. Nojiri and S.D. Odintsov, Int.J.Mod.Phys. A16 (2001) 1015, hepth/0009202.

[13] E. Elizalde, S. Naftulin and S.D. Odintsov, Phys.Rev. D49 (1994) 2852;

T. Chiba and M. Siino, Mod.Phys.Lett. A12 (1997) 709; S. Ichinose, hepth/9707025; W. Kummer, H. Liebl and D.V. Vassilevich, Mod.Phys.Lett. A12 (1997) 2683; J.S. Dowker, hep-th/9802029.

[14] S. Nojiri and S.D. Odintsov, Phys.Rev. D59 (1999) 044026, hepth/9804033.

[15] M. Cvetič and S. Gubser, JHEP 9904 (1999) 024. 
[16] A. Sen, Nucl.Phys. B475 562 (1996), hep-th/9605150; T. Banks, M.R. Douglas and N. Seiberg, Phys.Lett. B387 278 (1996), hep-th/9605199; O. Aharony, C. Sonnenstein, S. Yankielowicz and S. Theisen, Nucl.Phys. B493 177 (1997), hep-th/9611222; M.R. Douglas, D.A. Lowe, J.H. Schwarz, Phys.Lett. B394 297 (1997), hep-th/9612062.

[17] A. Fayyazuddin and M. Spalinski, Nucl.Phys. B535 219 (1998), hepth/9805096; O. Aharony, A. Fayyazuddin and J.M. Maldacena, JHEP 9807013 (1998), hep-th/9806159. 